\title{
Incompressible surfaces and Dehn Surgery on 1-bridge Knots in handlebodies
}

\author{
Ying-Qing Wu
}

Given a knot $K$ in a 3-manifold $M$, we use $N(K)$ to denote a regular neighborhood of $K$. Suppose $\gamma$ is a slope (i.e an isotopy class of essential simple closed curves) on $\partial N(K)$. The surgered manifold along $\gamma$ is denoted by $(H, K ; \gamma)$, which by definition is the manifold obtained by gluing a solid torus to $H-\operatorname{Int} N(K)$ so that $\gamma$ bounds a meridianal disk. We say that $M$ is $\partial$-reducible if $\partial M$ is compressible in $M$, and we call $\gamma$ a $\partial$-reducing slope of $K$ if $(H, K ; \gamma)$ is $\partial$-reducible. Since incompressible surfaces play an important rule in 3 -manifold theory, it is interesting to know what slopes of a given knot are $\partial$-reducing. In generic case there are at most three $\partial$-reducing slopes for a given knot [12], but there is no known algorithm to find these slopes. An exceptional case is when $M$ is a solid torus, which has been well studied by Berge, Gabai and Scharlemann $[1,4,5,10]$. It is now known that a knot in a solid torus has $\partial$-reducing slopes only if it is a 1-bridge braid. Moreover, all such knots and its corresponding $\partial$-reducing slopes are classified in [1]. For 1-bridge braids with small bridge width, a geometric method of detecting $\partial$-reducing slopes has also been given in [5]. It was conjectured that a similar result holds for handlebodies, i.e, if $K$ is a knot in a handlebody with $H-K \partial$-irreducible, then $K$ has $\partial$-reducing slopes only if $K$ is a 1-bridge knot (see below for definitions). One is referred to [13] for some discussion of this conjecture and related problems.

The main result of the present paper is to give an algorithm which will determine all $\partial$-reducing slopes for a given 1-bridge knot in a handlebody. Given a 1-bridge presentation of a knot $K$ in a handlebody $H$, the Main Algorithm in Section 7 will do the following.

(1). Determine if $K$ is disjoint from some compressing disk of $\partial H$. If it is, then $\partial H$ is compressible after all surgeries, so all slopes are $\partial$-reducing.

(2). If $K$ intersects all compressing disk of $\partial H$, determine if $K$ is isotopic to a simple closed curve on $\partial H$. If it is, then $\partial H$ is compressible in $(H, K ; \gamma)$ if and only if $\Delta\left(\gamma, \gamma_{0}\right) \leq 1$, where $\gamma_{0}$ is the the boundary of an annulus in $E(K)$ whose other boundary component is on $\partial H$.

\footnotetext{
${ }^{0}$ Mathematics Subject Classification: 57N10, 57M25, 57M50.
} 
(3). If the answer to 1 and 2 is negative, find three "candidate slopes". These are the only non meridional slopes which may be $\partial$-reducing.

(4). For each candidate slope $\gamma_{i}$, determine if $\left(H, K ; \gamma_{i}\right)$ is $\partial$-reducible.

The following is an outline of the algorithm.

Given a 1-bridge presentation $\alpha \cup \beta$ of $K$, and a cutting system $\mathcal{D}=D_{1} \cup \ldots \cup D_{h}$ of $H$ disjoint from $\beta$, let $B$ be the 3-ball obtained by cutting along $\mathcal{D}$. We can form a graph $\Gamma$ on $\partial B$ by letting $\partial \alpha$ and the copies of $D_{i}$ be the vertices, and $\alpha \cap \partial B$ be the edges. $\mathcal{D}$ can be modified so that $\Gamma$ has no "bad cut vertex" (Proposition 3.1). It can then be shown that $\partial H$ has a compressing disk disjoint from $K$ if and only if $\Gamma$ has a component disjoint from $\partial \alpha$. This completes (1) above. Also, if $\Gamma$ has two components, each containing a point of $\partial \alpha$, then $\partial H$ is incompressible after all nontrivial surgeries (Corollary 4.2). So we can assume that $\Gamma$ is connected.

The $\operatorname{arc} \beta$ is isotopic to any $\operatorname{arc} \delta$ on $\partial B$ with ends on $\partial \alpha$. The $\operatorname{arc} \delta$ is called a normal arc if, after shrinking $\delta$ to a point, the graph $(\Gamma \cup \delta) / \delta$ has no cut points. It is easy to find a normal arc (Proposition 5.1). The knot $K$ can be retrived from $\alpha \cup \delta$ by first pushing $\delta$ into Int $H$, then pushing $\alpha$ into Int $H$. This process determines an immersed annulus with one boundary $\partial^{\prime}$ on $\partial N(K)$, and the other one on $\alpha \cup \delta$. It can be shown that $K$ is isotopic to a simple closed curve if and only if $\delta$ is disjoint from $\alpha$, in which case $\gamma$ is a $\partial$-reducing slope if and only if $\Delta\left(\gamma, \partial^{\prime}\right) \leq 1$. This completes (2) above.

Now assume $\partial \cap \alpha \neq \emptyset$. There are three slopes $\gamma_{1}, \gamma_{2}, \gamma_{3}$ on $\partial N(K)$ which intersect the meridian just once, and intersect $\partial^{\prime}$ at most once. These are the candidate slopes. It will be shown (Theorem 6.2) that these are the only non meridianal slopes that can be $\partial$-reducing.

It remains to decide if $\gamma_{i}$ are $\partial$-reducing slopes. Theoretically it can be done by an algorithm of Haken using handle decomposition and normal surfaces (see [8]), but in practice that algorithm is quite sophisticated. We will do it as follows. Let $M$ be the handlebody $H-\operatorname{Int} N(\beta)$. It can be shown (Theorem 2.1) that each $\gamma_{i}$ corresponds to a curve $\gamma_{i}^{\prime}$ on $\partial M$ so that $\left(M, K ; \gamma_{i}\right)=M\left[\gamma_{i}^{\prime}\right]$, where $M\left[\gamma_{i}^{\prime}\right]$ denotes the manifold obtained by 2-handle addition on $M$ along $\gamma_{i}^{\prime}$. By Jaco's handle addition lemma, $M\left[\gamma_{i}^{\prime}\right]$ is $\partial$-reducible if and only if $\partial M-\gamma_{i}^{\prime}$ is compressible. Now a theorem of Starr (Theorem 1.2) can be used to determine if $\partial M-\gamma_{i}^{\prime}$ is compressible. This completes the algorithm.

The paper is organized as follows. In Section 1 and 2 we translate the problem of deciding whether a given boundary slope is $\partial$-reducing to a problem of deciding whether a certain curve on the boundary of a handlebody intersects all compressing disks, which can be accomplished by a result of Starr [11]. Sections $3-6$ will complete (1) - (3) above to find the candidate slopes. Theorem 4.1 is a generalization of a theorem of Menasco [9] and 
might be of independent interest. The Main Algorithm in Section 7 is a combination of the algorithms and theorems in these two steps. In Section 8 we give some final remarks. In particular, Theorem 8.3 has similar nature to the solid torus case, and is useful in practice.

Definitions and Conventions. One is referred to [6] for standard notations and conventions about 3-manifold. In particular, we will always assume that two submanifolds are in general position.

We use $H$ to denote a handlebody, and use $K$ to denote a knot in $H$. K is called a 1-bridge knot if it is isotopic to some curve $\alpha \cup \beta$, where $\alpha$ is an arc on $\partial H$, and $\beta$ is a proper arc in $H$ which is rel $\partial \beta$ isotopic to some $\operatorname{arc} \beta^{\prime}$ on $\partial H$. In this case $\alpha \cup \beta$ is called a 1-bridge presentation of $K$. Note that the two $\operatorname{arcs} \alpha$ and $\beta^{\prime}$ may intersect each other, but they have no self intersections.

Given a curve $\gamma$ on the boundary of a 3 -manifold $M$, we can glue a $D^{2} \times I$ to $M$ so that $\partial D^{2} \times I$ is glued to a regular neighborhood of $\gamma$ on $\partial M$. This process is called a 2-handle addition, and the manifold so obtained is denoted by $M[\gamma]$.

\section{Compressibility of $\partial H-\gamma$}

Consider a set of simple closed curves $\gamma$ on the boundary of a handlebody $H$. We need an algorithm to determine if $\partial H-\gamma$ is compressible. The proof of Proposition 1.1 will produce a cutting system so that the corresponding graph is either disconnected or 2-connected (see below for definitions.) Corollary 1.3 then asserts that $\partial H-\gamma$ is compressible if and only if the graph is disconnected. Theorem 1.2 is due to Edith Starr [11]. We give a proof here for the convenience of the readers.

Recall that a set of disks $\mathcal{D}=D_{1} \cup \ldots \cup D_{g}$ is a cutting system of $H$ if after cutting along $\mathcal{D}$, the manifold $B=H-\operatorname{Int} N(\mathcal{D})$ is a 3 -ball. Each $D_{i}$ gives rise to two disks $D_{i}^{\prime}$ and $D_{i}^{\prime \prime}$ on $\partial B$, which we will consider as "fat" vertices. Each arc component of $\gamma \cap \partial B$ is considered an edge. If $\gamma \cap \partial B$ has circle components, add a vertex to each of them. We thus obtain a planar graph on the sphere $\partial B$, denoted by $\Gamma=\Gamma(\mathcal{D})$.

Some convention is in order. Since we are dealing with graphs with fat vertices, a loop is actually an arc $e$ on the sphere $\partial B$ which has both ends on a fat vertex $v$. By a disk on $\partial B$ bounded by $e$ we mean a disk $D$ on $\partial B$ which is bounded by $e$ and a boundary arc of $v$, such that $D$ is disjoint from the interior of the fat vertex $e$.

A vertex $v$ on a graph $\Gamma$ is called a cut vertex if $\Gamma-v$ has more components than $\Gamma$. In this case there is a circle $C$ on $\partial B$, called a cut circle, which intersects $\Gamma$ only at $v$, and both sides of $C$ has edges of $\Gamma$ incident to $v$. $\Gamma$ is called 2-connected if it is connected and has no cut vertices. 
Proposition 1.1 Given curves $\gamma$ on $\partial H$, there is a cutting set $\mathcal{D}$ so that $\Gamma(\mathcal{D})$ has no cut vertices. Thus $\Gamma(\mathcal{D})$ is either disconnected or 2-connected.

Proof. Start with an arbitrary cutting system $\mathcal{D}^{\prime}$. Suppose $\Gamma\left(\mathcal{D}^{\prime}\right)$ is a cut vertex, say $D_{1}^{\prime}$. Let $C$ be the corresponding cut circle, which is an arc with ends on $\partial D_{1}^{\prime}$. The two ends of $C$ cut $\partial D_{1}^{\prime}$ into two $\operatorname{arcs} e_{1}, e_{2}$, and we have two circles $C_{1}=C \cup e_{1}$ and $C_{2}=C \cup e_{2}$, each having less intersection with $\gamma$ than $\partial D_{1}^{\prime}$. Exactly one of the $C_{i}$, say $C_{1}$, separates the two disks $D_{1}^{\prime}$ and $D_{1}^{\prime \prime}$. Let $D_{0}$ be a disk in $B$ bounded by $C_{1}$. Then $\mathcal{D}^{\prime \prime}=D_{0} \cup D_{2} \cup \ldots \cup D_{g}$ is a cutting set with $\left|\mathcal{D}^{\prime \prime} \cap \gamma\right|<\left|\mathcal{D}^{\prime} \cap \gamma\right|$. Now consider $\Gamma\left(\mathcal{D}^{\prime \prime}\right)$ and repeat the above process. q.e.d

Theorem 1.2 (Starr [11]) $\partial H-\gamma$ is incompressible if and only if there is a cutting system $\mathcal{D}$ such that $\Gamma(\mathcal{D})$ is 2-connected.

Proof. Suppose $\partial H-\gamma$ is compressible with $E$ a compressing disk such that $|E \cap \mathcal{D}|$ is minimal. If $\partial E$ is disjoint from $\mathcal{D}$ then $\partial E$ is a circle on $\partial B$ which separates $\Gamma$, so $\Gamma$ is disconnected. If $\partial E \cap \mathcal{D} \neq \emptyset$ then $E \cap \mathcal{D}$ has some arc components. An outermost arc on $E$ cuts off an arc $e$ of $\partial E$ which has both ends on the same fat vertex of $\Gamma$ and has interior disjoint from $\Gamma$. If $e$ bounds a disk on $\partial B$ with interior disjoint from $\Gamma$ then one can use this disk to isotop $E$ and get another compressing disk $E^{\prime}$ with $\left|E^{\prime} \cap \mathcal{D}\right|<|E \cap \mathcal{D}|$, contradicting the choice of $E$. Hence both sides of $e$ intersects $\Gamma$, so either $\Gamma$ is disconnected, or $v$ is a cut vertex. This proves the sufficiency.

Now suppose $\partial H-\gamma$ is incompressible. Let $\mathcal{D}$ be the cutting system obtained by Propsition 1.1. If $\Gamma(\mathcal{D})$ is disconnected, a separating circle would bound a disk which would be a compressing disk of $\partial-\gamma$, which is absurd. Therefore $\Gamma(\mathcal{D})$ is 2-connected. q.e.d

Corollary 1.3 Let $\mathcal{D}$ be a cutting system so that $\Gamma(\mathcal{D})$ has no cut vertices. Then $\partial H-\gamma$ is incompressible if and only if $\Gamma(\mathcal{D})$ is connected.

$$
\text { q.e.d }
$$

A more general result is true. Suppose $\mathcal{D}$ is a set of disks in $H$ cutting $H$ into a union of 3-balls $B_{1} \cup \ldots \cup B_{k}$. Construct a graph $\Gamma=\Gamma(\mathcal{D})$ as above. Let $\Gamma_{i}$ be the part of $\Gamma$ on $\partial B_{i}$. We will need the following result for the proof of Theorem 6.2.

Corollary 1.4 If $\Gamma_{i}$ is 2-connected for all $i$, then $\partial H-\gamma$ is incompressible.

Proof. The proof is essentially the same as that for the sufficiency of Theorem 1.2. q.e.d 


\section{$2 \quad \partial$-reducibility of a given surgery}

Suppose $K$ is a knot in a handlebody $H$ with 1-bridge presentation $\alpha \cup \beta$. Let $M$ be the handlebody $H-\operatorname{Int} N(\beta)$. Consider the punctured torus $P=\partial N(\beta) \cup N_{\partial}(\alpha)$, where $N_{\partial}(\alpha)$ denotes a regular neighborhood of $\alpha$ on $\partial M$. It is easy to see that when attaching a 2-handle to $M$ along the curve $\partial P$, we get a manifold $X=M[\partial P]$, which is homeomorphic to the knot exterior $H-\operatorname{Int} N(K)$. We identify $X$ with $H-\operatorname{Int} N(K)$. The punctured torus $P$ lies on $\partial N(K)$, so any slope $\gamma$ on $\partial N(K)$ can be isotoped into $P$.

Theorem 2.1 With the above identification, the surgered manifold $(H, K ; \gamma)$ is homeomorphic to $M[\gamma]$.

Proof. By definition, $(H, K ; \gamma)$ is obtained from $X$ by attaching a solid torus $V$ to $\partial N(K)$ so that $\gamma$ bounds a meridian $D$ of $V$. This can be done in two steps: Adding a neighborhood $N(D)$ of $D$, then adding the rest part. The first step is a 2-handle addition along the curve $\gamma$, while the second is simply gluing a 3-ball to the manifold along its boundary, called "adding a 3-handle". If we use $B$ to denote the 3 -handle, then the above can be written as

$$
(H, K ; \gamma)=X[\gamma] \cup B=M[\partial P][\gamma] \cup B
$$

Since $\partial P$ and $\gamma$ are disjoint, we can switch the order of the two 2-handle additions, and get

$$
(H, K ; \gamma)=M[\gamma][\partial P] \cup B
$$

Notice that the 2-handle added along $\partial P$ intersects the 3 -handle at a disk, so they cancel each other. Therefore $(H, K ; \gamma)$ is homeomorphic to $M[\gamma]$ q.e.d

Corollary $2.2(H, K ; \gamma)$ is $\partial$-reducible if and only if $\partial M-\gamma$ is compressible.

Proof. Since $M$ is a handlebody, $\partial M$ is compressible. The result now follows from the above theorem and Jaco's handle addition lemma [7, Lemma 1]. q.e.d

Given a slope $\gamma$ on $\partial N(K)$, Corollary 2.2 and Proposition 1.1 can be used to determine if $\partial H$ is compressible in $(H, K ; \gamma)$. The remaining task is to determine a finite set of "candidate slopes" so that surgeries along all other slopes produce $\partial$-irreducible manifolds. We can then use the above to check this finite set of slopes and see if there are any slopes along which surgeries produce $\partial$-reducible manifolds. 


\section{$3 \quad$ Find a good cutting system}

Suppose $\mathcal{D}=D_{1} \cup \ldots \cup D_{g}$ is a cutting system. Let $B=H-\operatorname{Int} N(\mathcal{D})$ be the 3 -manifold obtained by cutting $H$ along $\mathcal{D}$. As in section 1 , each $D_{i}$ determines two fat vertices $D_{i}^{\prime}$ and $D_{i}^{\prime \prime}$ on $\partial B$. Consider the two ends of $\alpha$ also as vertices, and consider the $\operatorname{arcs}$ in $\alpha \cap B$ as edges. Then we get a graph $\Gamma=\Gamma(\alpha, \mathcal{D})$.

Definition. Let $\Gamma=\Gamma(\alpha, \mathcal{D})$ be the graph corresponding to a cutting system $\mathcal{D}$. A cut vertex $v$ of $\Gamma$ is called a bad cut vertex if there is a cut circle $C$ for $v$ such that the two ends of $\alpha$ lie on the same disk on $\partial B$ bounded by $C$. Similarly, a separating circle $C$ for $\Gamma$ is called a bad separating circle if the two ends of $\alpha$ are on the same disk bounded by $C$. A cutting system $\mathcal{D}$ is called a fair cutting system if it is disjoint from $\beta$, and $\Gamma(\alpha, \mathcal{D})$ has no bad cut vertex. If in addition there is no bad separating circles for $\Gamma(\alpha, \mathcal{D})$, then $\mathcal{D}$ is called a good cutting system.

Proposition 3.1 There exists a fair cutting system $\mathcal{D}$.

Proof. Start with an arbitrary cutting system $\mathcal{D}$ which is disjoint from $\beta$. If $\Gamma(\alpha, \mathcal{D})$ has no bad cut vertex we are done. Assume $v$ is a bad cut vertex, and let $e$ be the corresponding cut circle. Without loss of generality we may assume that $v=D_{1}^{\prime}$. Then $\partial e$ cuts $\partial D_{1}^{\prime}$ into two edges $e_{1}$ and $e_{2}$. Let $C_{i}=e \cup e_{i}$. One of the $C_{i}$, say $C_{1}$, separates $D_{1}^{\prime}$ from $D_{1}^{\prime \prime}$. Since $C_{1}$ does not separates the two ends of $\alpha$, it bounds a disk $D_{0}$ which is disjoint from $\beta$. Replace $D_{1}$ by $D_{0}$ to get a new cutting system $\mathcal{D}^{\prime}$ which is still disjoint from $\beta$. If $\Gamma\left(\alpha, \mathcal{D}^{\prime}\right)$ has bad cut vertex, repeat the process. Since $\left|\mathcal{D}^{\prime} \cap \alpha\right|<|\mathcal{D} \cap \alpha|$, the process will eventually stop, and produce a cutting system $\mathcal{D}^{\prime \prime}$ such that $\Gamma\left(\alpha, \mathcal{D}^{\prime \prime}\right)$ has no bad cut vertex. q.e.d

We remark that in general there is no cutting system $\mathcal{D}$ which is disjoint from $\beta$ and yet has no cut vertices.

Lemma 3.2 Suppose $\alpha \cup \beta$ is a 1-bridge presentation of $K$. Then $\partial H$ is compressible in $H-K$ if and only if $\partial H-\alpha$ is compressible in $H-(\alpha \cup \beta)$.

Proof. Consider $M=H-\operatorname{Int} N(\beta)$ and $P=\partial N(\beta) \cup N_{\partial}(\alpha)$. We identify $H-\operatorname{Int} N(K)$ with $M[\partial P]$, as in Section 2. Clearly, there is a homeomorphism $M-P \cong H-(\alpha \cup \beta)$, which sends the surface $\partial M-P$ to $\partial H-\alpha$.

If $\partial M-P$ is compressible in $M-P$, a compressing disk will either be a compressing disk of $\partial H$ in $H-\operatorname{Int} N(K)=M[\partial P]$, or it cuts off a 3-ball of $H$ containing $K$. In either case $\partial H$ is compressible in $H-K$.

Now suppose $\partial M-P$ is incompressible. In particular $\partial P$ does not bound disk in $M$. Since $P$ is a punctured torus, $\partial P$ is coplanar to any essential curve in $P$, so no essential curve 
in $P$ bounds any compressing disk. In other words, $P$ is also incompressible. Therefore $\partial M-\partial P$ is incompressible in $M$. Since $\partial M$ is compressible, by Jaco's handle addition lemma [7], the manifold $M[\partial P]=H-\operatorname{Int} N(K)$ has incompressible boundary. $\quad$ q.e.d

Theorem 3.3 $\partial H$ is incompressible in $H-K$ if and only if there exists a good cutting system.

Proof. The proof is similar to that of Theorem 1.2. Suppose $\mathcal{D}$ is a good cutting system. If $\partial H$ is compressible in $H-K$ then by Lemma 3.2 there is a compressing disk $E$ of $\partial H-\alpha$ in $H-(\alpha \cup \beta)$. Isotop $E$ so that $|E \cap \mathcal{D}|$ is minimal. If $E \cap \mathcal{D}=\emptyset$, then the curve $\partial E$ is a circle in $\partial B$ disjoint from $\Gamma$, and both disks of $\partial B$ bounded by $\partial E$ intersect $\Gamma$. Moreover, since $E$ is disjoint from $\beta$, the two points $\partial \beta=\partial \alpha$ lie on the same side of $\partial E$. Thus $\partial E$ is a bad separating circle for $\Gamma$, contradicting the assumption that $\mathcal{D}$ is a good cutting system. So assume $E \cap \mathcal{D} \neq \emptyset$. Then an arc of $E \cap \mathcal{D}$ which is outermost on $E$ cuts off a disk $E^{\prime}$ in $B$ whose boundary $\partial E^{\prime}$ intersects $\Gamma$ just at a fat vertex. If one component of $\partial B-\partial E^{\prime}$ is disjoint from $\Gamma$, one can isotop $\mathcal{D}$ to reduce $|E \cap \mathcal{D}|$, which contradicts the choice of $\mathcal{D}$. Hence both sides of $\partial E^{\prime}$ contain parts of $\Gamma$. Again, the two ends of $\alpha$ lie on same side of $\partial E^{\prime}$ because $E^{\prime} \cap \beta=\emptyset$. Hence either the vertex at which $\partial E^{\prime}$ is based at is a bad cutting vertex, or $\partial E^{\prime}$ can be pushed off the vertex to become a bad separating circle for $\Gamma$. Both cases contradict the assumption that $\mathcal{D}$ is a good cutting system, completing the proof of sufficiency.

For necessity, let $\mathcal{D}$ be a fair cutting system produced by Proposition 3.1. Then $\Gamma(\alpha, \mathcal{D})$ has no bad cut vertex. If there is a bad separating circle $C$, then $C$ bounds a disk $E$ in $B$ which is disjoint from $\Gamma$ and $\beta$, so $E$ is a compressing disk of $\partial H-\alpha$ in $H-\alpha \cup \beta$. By Lemma $3.2 \partial H$ is compressible in $H-K$.

q.e.d

Corollary 3.4 Let $\mathcal{D}$ be a cutting system produced by Proposition 3.1. Then $\partial H$ is incompressible in $H-K$ if and only if $\mathcal{D}$ is a good cutting system.

q.e.d

Note that if $\mathcal{D}$ is obtained by Proposition 3.1, then it is fair, so it is good if and only if each component of $\Gamma(\alpha, \mathcal{D})$ contains some ends of $\alpha$.

\section{If $\Gamma(\alpha, \mathcal{D})$ is disconnected}

We now assume that $\partial H$ is incompressible in $H-K$. By Corollary 3.4 and Proposition 3.1 we can find a good cutting system. This section deals with the special case that $\Gamma(\alpha, \mathcal{D})$ is disconnected. We will show that in this case all nontrivial surgeries on $K$ produce $\partial$ irreducible manifolds. This will follow from Theorem 4.1, which generalize a theorem of Menasco [9]. 
Recall that a curve $C_{1}$ on a surface $F$ is called coplanar to another curve $C_{2}$ if either they are parallel or $C_{1}$ bounds a once punctured torus which contains $C_{2}$ as a nonseparating curve. If $F$ is the boundary of a 3 -manifold and $C_{2}$ bounds a compressing disk, then so does any curve coplanar to $C_{2}$. Note also that if $C_{1}, C_{2}$ are nonseparating, then $C_{1}$ is coplanar to $C_{2}$ if and only if it is parallel to $C_{2}$.

Theorem 4.1 Suppose $K$ is a knot in a 3-manifold $M$ such that $\partial M$ is incompressible in $M-K$. Suppose $D_{1}, D_{2}$ are compressing disks of $\partial M$ in $M$ such that $D_{1}$ intersects $K$ at one point, and $\partial D_{1}$ is not coplanar to $\partial D_{2}$. Then $\partial M$ is incompressible in all manifolds resulted from nontrivial surgeries on $K$.

Proof. Let $X$ be the knot exterior $M-\operatorname{Int} N(K)$. Let $A$ be the annulus $D_{1} \cap X$. It is incompressible in $X$, otherwise $\partial D_{1}$ would bounds a compressing disk of $\partial M$ in $X$, contradicting our assumption. By [3, Theorem 2.4.3], either $X$ is (torus $\times I$ ), or $\partial M$ remains incompressible in all $(M, K ; \gamma)$ with $\Delta(\gamma, m) \geq 2$, where $m$ is the meridian slope of $K$. The first case is impossible because then $\partial D_{2}$ would be parallel to $\partial D_{1}$.

Now assume $\Delta(\gamma, m)=1$. We have $(M, K ; \gamma)=X \cup V$, where $V$ is the attached solid torus. Let $X^{\prime}=X-\operatorname{Int} N(A)$. Then

$$
X \cup V=(X-\operatorname{Int} N(A)) \cup N(A) \cup V=X^{\prime} \cup(N(A) \cup V)
$$

Since the inner boundary of $A$ intersects the meridian of $V$ just once, $V^{\prime}=N(A) \cup V$ is a solid torus, whose frontier in $X \cup V$ is an annulus which is the neighborhood of a longitude of $V^{\prime}$. Therefore, gluing $V^{\prime}$ to $X^{\prime}$ produces a manifold homeomorphic to $X^{\prime}$. In other words, the surgered manifold $(M, K ; \gamma)$ is homeomorphic to the manifold $X^{\prime}$ obtained by cutting $X$ along the annulus $A$.

Denote by $\gamma$ the central curve of the annulus $\partial N(K) \cap X^{\prime}$. Notice that $\partial X^{\prime}-\gamma$ is incompressible, for a compressing disk $E$ could be isotoped to have boundary lying on $\partial X^{\prime} \cap \partial M$, so it would be a compressing disk of $\partial M$ in $X=M-\operatorname{Int} N(K)$, contradicting our assumption. If $\partial(M, K ; \gamma)=\partial X^{\prime}$ were compressible, by Jaco's Handle Addition Lemma [7] the manifold $X^{\prime}[\gamma]$ obtained by attaching a 2-handle to $X^{\prime}$ along the curve $\gamma$ would have incompressible boundary. But this is impossible because one can see that $X^{\prime}[\gamma]$ is homeomorphic to $M-\operatorname{Int} N\left(D_{1}\right)$, and since $\partial D_{2}$ is not coplanar to $\partial D_{1}, D_{2}$ is a compressing disk of the boundary of $M-\operatorname{Int} N\left(D_{1}\right)$.

q.e.d

Corollary 4.2 Let $\mathcal{D}$ be a good cutting system, and $\Gamma(\alpha, \mathcal{D})$ the corresponding graph. If $\Gamma(\alpha, \mathcal{D})$ is disconnected, then either $H$ is a solid torus with $K$ as a central curve, or all nontrivial surgeries on $K$ produce $\partial$-irreducible manifolds. 
Proof. If $\Gamma(\alpha, \mathcal{D})$ is disconnected, there is a curve $C$ on $\partial B$ which separates $\Gamma(\alpha, \mathcal{D})$. Since $\mathcal{D}$ is a good cutting system, the two ends of $\alpha$ lie on different sides of $C$, so $C$ bounds a disk $D$ in $B$ which is disjoint from $\alpha$ and intersects $\beta$ at one point. Such a disk is a compressing disk of $\partial H$ intersecting $K$ just once, so the result follows from [4, Theorem 1.1] if $H$ has genus one. When $H$ has genus greater than one, $H-\operatorname{Int} N(D)$ is a handlebody, so we can find another compressing disk $D_{2}$ of $\partial H$ which is nonseparating and is not parallel to $D$. In this case the result follows from Theorem 4.1.

q.e.d

\section{Find a normal connecting arc}

In this section we assume $\mathcal{D}$ is a good cutting system such that $\Gamma=\Gamma(\alpha, \mathcal{D})$ is connected. Define a connecting arc to be an $\operatorname{arc} \delta$ on $\partial B$ which has ends on $\partial \alpha$ and is disjoint from the fat vertices. It is called a normal connecting arc if after shrinking $\delta$ to a point, the graph $\Gamma_{\delta}=(\Gamma \cup \delta) / \delta$ has no cut vertices. We use $[\delta]$ to denote the vertex in $\Gamma_{\delta}$ which is the image of $\delta$.

Proposition 5.1 Given a good cutting system $\mathcal{D}$ with $\Gamma(\alpha, \mathcal{D})$ connected, there is a normal connecting arc.

Proof. Choose an arbitrary connecting $\operatorname{arc} \delta$ and consider $\Gamma_{\delta}$. Since $\Gamma$ is connected and has no bad cut vertex, it is easy to see that no fat vertex can be a cut vertex of $\Gamma_{\delta}$. If $[\delta]$ is not a cut vertex either then we are done. Otherwise, let $C$ be a circle on $\partial B / \delta$ intersecting $\Gamma_{\delta}$ at $[\delta]$ such that both sides of $C$ intersects $\Gamma_{\delta}$. Such circle corresponds to an $\operatorname{arc} C^{\prime}$ on $\partial B$ which is disjoint from $\Gamma$ and has both ends on the $\operatorname{arc} \delta$. Let $e$ be the segment of $\delta$ between the two ends of $C^{\prime}$. If $e \cap \Gamma=\emptyset, C^{\prime} \cup e$ would be a circle which separates $\Gamma$, contradicting the connectivity of $\Gamma$. Hence when replacing $e$ by $C^{\prime}$ we get another connecting arc $\delta^{\prime}$ with $\left|\delta^{\prime} \cap \Gamma\right|<|\delta \cap \Gamma|$. Repeat this process, we will eventually obtain a normal connecting arc. q.e.d

Corollary 5.2 If $\delta$ is a connecting arc of least intersection with $\Gamma$, then $\delta$ is normal. q.e.d

\section{Find candidate slopes}

Since $\beta$ is an unknotted arc in $B$, for any connecting $\operatorname{arc} \delta$ on $\partial B, \beta \cup \delta$ bounds an embedded disk $D$. If $\delta$ is disjoint from $\operatorname{Int}(\alpha)$, then $K$ is isotopic to the simple closed curve $\delta \cup \beta$ on $\partial H$, in which case there are infinitely many $\partial$-reducing surgeries, and the slopes are known $[3$, Theorem 2.4.3]. So we need only deal with the case that $\delta \cap \operatorname{Int} \alpha \neq \emptyset$. First we have 
Lemma 6.1 Let $\mathcal{D}$ be a good cutting system, and let $\delta$ be a normal connecting arc. Then one of the following holds.

(1) $\delta \cap \operatorname{Int}(\alpha)=\emptyset$.

(2) There is a compressing disk of $\partial H$ intersecting $K$ only once.

(3) There is no incompressible annulus in $X=H-\operatorname{Int} N(K)$ with one boundary component on each of $\partial H$ and $\partial N(K)$.

Proof. Assuming (3) is false, let $A$ be an incompressible annulus with one boundary on each of $\partial H$ and $\partial N(K)$. Let $l$ be the slope $A \cap \partial N(K)$. Since $H=(H, K ; m)$ is $\partial$-reducible, by $[3$, Theorem 2.4.3] we must have $\Delta(m, l) \leq 1$. If $l=m$, then $A$ extends to a disk in $H$ intersecting $K$ just once, giving conclusion (2). So we can assume $\Delta(l, m)=1$. By $[3$, Theorem 2.4.3] any slope $\gamma$ with $\Delta(\gamma, l)=1$ is a $\partial$-reducing slope. In particular there is a slope $\gamma$ on $\partial N(K)$ with $\Delta(m, \gamma) \geq 2$ such that $(H, K ; \gamma)$ is $\partial$-reducible. But according to Proposition 1 of [12] and its proof, this implies that $\beta$ is rel $\partial \beta$ isotopic to an arc $\beta^{\prime}$ on $\partial H$ with $\beta^{\prime} \cap \operatorname{Int}(\alpha)=\emptyset$. Let $E$ be a disk bounded by $\beta \cup \beta^{\prime}$. By the same proof as that for Theorem 3.3, one can show that $E$ can be made disjoint from $\mathcal{D}$. Therefore $\beta^{\prime}$ is a connecting arc. After shrinking $\delta$ to a point, $\beta^{\prime}$ becomes some circles in $\Gamma_{\delta}$. If (1) is false, then $\beta^{\prime} \cup \delta$ intersects $\operatorname{Int}(\alpha)$, so at least one of these circles is a cut circle for $[\delta]$, contradicting the assumption that $\delta$ is a normal connecting arc. q.e.d

Now consider the manifold $M=H-\operatorname{Int} N(\beta)$, and the punctured torus $P=\partial N(\beta) \cup$ $N_{\partial}(\alpha)$ as defined in Section 2. Let $D_{g+1}=D \cap M$, where $D$ is a disk bounded by $\beta \cup \delta$. Then $\partial D_{g+1}$ intersects $P$ at an arc and cuts $P$ into an annulus. There are 3 simple closed curves $\gamma_{1}, \gamma_{2}, \gamma_{3}$ on $P$ which runs through $N_{\partial}(\alpha)$ only once and intersects $\partial D_{g+1}$ at most once. Call these the candidate slopes (associated to $\delta$ ).

Theorem 6.2 Suppose $\mathcal{D}$ is a good cutting system, and suppose $\delta$ is a normal connecting arc on $\partial B$ with $\delta \cap \operatorname{Int}(\alpha) \neq \emptyset$. Let $\gamma$ be a slope on $P$ which is not a meridian of $\beta$. Then $\partial M-\gamma$ is incompressible unless $\gamma$ is one of the candidate slopes associated to $\delta$.

Proof. Recall that by Corollary $2.2 \partial-\gamma$ is compressible if and only if $\partial H$ is compressible in $(H, K ; \gamma)$. Let $\gamma$ be a non-meridianal slope on $\partial N(K)$ such that $\partial H$ is compressible in $(H, K ; \gamma)$. Apply Lemma 6.1. Our assumption rules out conclusion (1). Also, Corollary 4.2 says that (2) is false. Therefore (3) holds. By Theorem 1 of [12], we must have $\Delta(\gamma, m)=1$. In other words, $\gamma$ can be written as $\gamma=\alpha^{\prime} \cup \tau$, where $\alpha^{\prime}$ is the part of $\alpha$ on $\partial M$ (i.e, $\alpha$ with the two small $\operatorname{arcs} \alpha \cap \operatorname{Int} N(\beta)$ removed), and $\tau$ is an arc on $\partial N(\beta)$, isotoped to have least intersection with $\partial D_{g+1}$. 
Let $D_{g+2}$ be a disk in $H$ which is disjoint from $\beta$, such that $\partial D_{g+2}$ is the boundary of a regular neighborhood of the $\operatorname{arc} \delta$. The set $\mathcal{D}^{\prime}=\mathcal{D} \cup D_{g+1} \cup D_{g+2}$ cuts $M$ into two 3-balls $B_{1}$ and $B_{2}$. We assume $B_{2}$ is the one that contains the fat vertices $D_{g+1}^{\prime}$ and $D_{g+1}^{\prime \prime}$.

Consider the graph $\Gamma\left(\mathcal{D}^{\prime}, \gamma\right)$ which has fat vertices as above, and has $\gamma \cap\left(\partial B_{1} \cup \partial B_{2}\right)$ as edges. Let $\Gamma_{i}=\Gamma \cap \partial B_{i}$. It is easy to see that $\Gamma_{1}$ is isomorphic to the graph $\Gamma_{\delta}$ defined in Section 5 , so it is 2-connected because we have assumed that $\delta$ is a normal connecting arc.

Now consider the graph $\Gamma_{2}$. It has three vertices: $D_{g+1}^{\prime}, D_{g+1}^{\prime \prime}$, and $D_{g+2}^{\prime}$, say. Since $\delta \cap(\alpha) \neq \emptyset$, there are edges of $\Gamma_{2}$ connecting $D_{g+2}^{\prime}$ to both $D_{g+1}^{\prime}$ and $D_{g+1}^{\prime \prime}$. Since $\tau \cap \partial D_{g+1}$ is assumed minimal, there are no loops in $\Gamma_{2}$. Moreover, if $\gamma$ is not one of the candidate slopes, then $\left|\tau \cap \partial D_{g+1}\right|=\left|\gamma \cap \partial D_{g+1}\right| \geq 2$, so there is at least one edge of $\Gamma_{2}$ which connects the two vertices $D_{g+1}^{\prime}$ and $D_{g+1}^{\prime \prime}$. Hence the graph $\Gamma_{2}$ is also 2-connected. By Corollary 1.4, $\partial M-\gamma$ is incompressible.

q.e.d

\section{The main algorithm}

The Main Algorithm is a combination of the results in the previous sections. It will determine whether $\partial H$ is compressible in $H-K$, whether $K$ is boundary parallel, and determine all $\partial$-reducing slopes if there is any.

Main Algorithm. Given a 1-bridge presentation $\alpha \cup \beta$ of a knot $K$ in a handlebody $H$, find all $\partial$-reducing slopes on $\partial N(K)$.

Step 1. Use Proposition 3.1 to find a fair cutting system. By Corollary 3.4, if there is a bad separating circle for $\Gamma(\alpha, \mathcal{D})$ then $\partial H$ is compressible in $H-K$, so all slopes of $\partial N(K)$ are $\partial$-reducing. Otherwise, $\mathcal{D}$ is a good cutting system.

Step 2. If $\Gamma(\alpha, \mathcal{D})$ is disconnected, then there is no $\partial$-reducing slopes other than the median $m$ of $K$ (Corollary 4.2). Otherwise, use Proposition 5.1 to find a normal connecting $\operatorname{arc} \delta$. If $\delta$ is disjoint from $\operatorname{Int}(\alpha)$ then $K$ is isotopic to the simple closed curve $\alpha \cup \delta$ on $\partial H$, so a slope $\gamma$ is $\partial$-reducing if and only if $\Delta(\gamma, l) \leq 1$, where $l$ is the slope determined by an annulus bounded by $K$ and $\alpha \cup \delta$ [3, Theorem 2.4.3]. Otherwise, let $\gamma_{1}, \gamma_{2}, \gamma_{3}$ be the 3 candidate slopes defined in Section 6.

Step 3. Let $M$ and $P$ be as in Section 2. Use Proposition 1.1 and Corollary 1.3 to determine if $\partial M-\gamma_{i}$ is compressible for $i=1,2,3$. Corollary 2.2 and Theorems 6.1 say that $(H, K ; \gamma)$ is $\partial$-reducible if and only if $\gamma$ is one of the $\gamma_{i}^{\prime}$ 's and $\partial M-\gamma$ is compressible. q.e.d 


\section{Remarks and related results}

Here are some related results. Sometimes they will provide quick decision about whether $K$ admits $\partial$-reducing surgeries.

8.1. A 1-bridge knot $K$ is called to have width 1 if the bridge $\beta$ is rel $\partial \beta$ isotopic to some $\operatorname{arc} \beta^{\prime}$ on $\partial H$ which intersects $\operatorname{Int}(\alpha)$ just once. One can show that if $K$ has width 1 then there is a nontrivial surgery on $K$ that produces a handlebody.

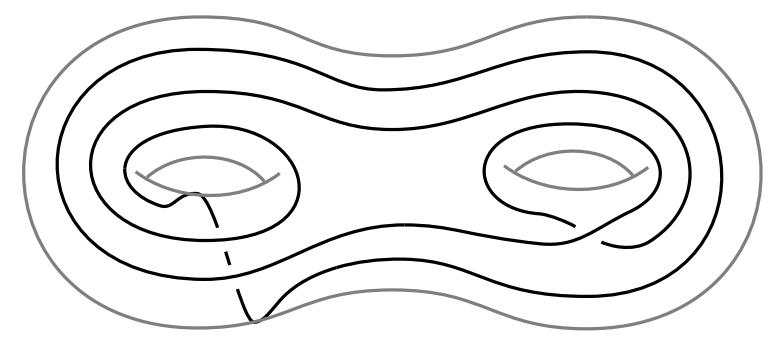

Figure 1

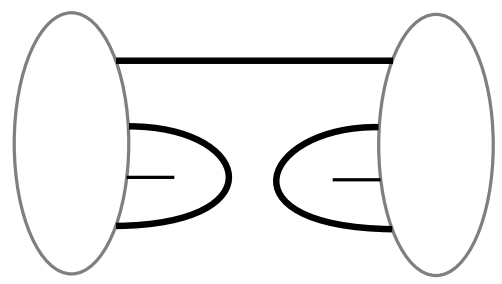

Figure 2

8.2. The knot shown in Figure 1 is a width 1 knot in a genus 2 handlebody. It is actually width 1 in two different ways. One can show that there are three different surgeries (including the trivial one) that produce handlebodies. By changing the way the curve winding around the left hand side handle one can get many different knots with this property. This should be compare with the solid torus case. It has been shown by Berge [1] that there is exactly one nontrivial knot which admits three different surgeries producing solid tori.

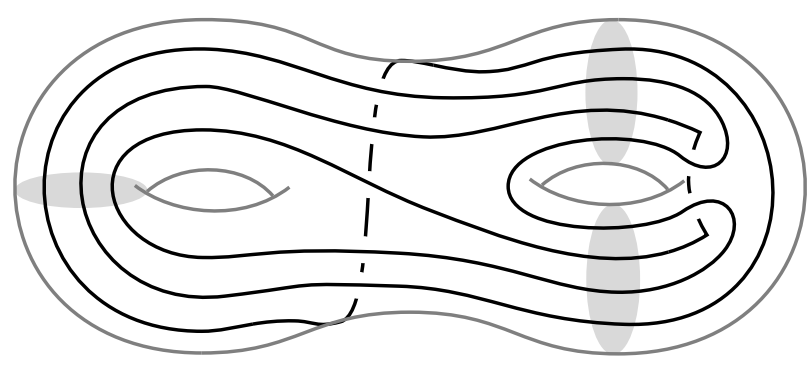

Figure 3

8.3. A consequence of the results of Berge [1], Gabai [4] and Scharlemann [10] is that if $K$ is a 1-bridge knot in a solid torus but is not a closed braid, then there is no nontrivial 
$\partial$-reducing surgeries. By cutting along a good disk one gets a graph with two fat vertices which looks like that in Figure 2, where the thick line represents a nonempty set of parallel lines. The following is a similar result for handlebodies. The proof is similar to that of Theorem 6.2 and is omitted. One can use the theorem to show that the knot in Figure 3 admits no nontrivial $\partial$-reducing surgeries. The cutting disks are shown in the picture.

Theorem. Suppose $\mathcal{D}$ is a set of disks disjoint from $\beta$, cutting $H$ into two 3-balls $B_{1}$ and $B_{2}$. Let $\Gamma(\alpha, \mathcal{D})$ be defined as in Section 3, and let $\Gamma_{i}=\Gamma(\alpha, \mathcal{D}) \cap B_{i}$. If $\Gamma_{1}$ is 2-connected, and $\Gamma_{2}$ is the graph in Figure 2 , then $K$ admits no nontrivial $\partial$-reducing surgeries.

\section{References}

[1] J. Berge: The knots in $D^{2} \times S^{1}$ with nontrivial Dehn surgery yielding $D^{2} \times S^{1}$, Topology Appl. 38 (1991) 1-19.

[2] J. Berge: Knots in handlebodies which can be surgered to produce handlebodies, preprint.

[3] M. Culler, C. Gordon, J. Luecke and P. Shalen: Dehn surgery on knots, Ann. Math. 125 (1987) $237-300$.

[4] D. Gabai: On 1-bridge braids in solid tori, Topology 28 (1989) 1-6.

[5] D. Gabai: 1-bridge braids in solid tori, Topology Appl. 37 (1990) 221-235.

[6] J. Hempel: 3-manifolds (Ann. Math. Stud., Vol. 86) Princeton NJ: Princeton University Press, 1976.

[7] W. Jaco: Adding a 2-handle to 3-manifolds: An application to Property R, Proc. Amer. Math. Soc. 92 (1984) 288-292.

[8] W. Jaco and U. Oertel: An algorithm to decide if a 3-manifold is a Haken manifold, Topology 23 (1994) 195-209.

[9] W. Menasco: Closed incompressible surfaces in alternating knot and link complements, Topology 23 (1984) 37-44.

[10] M. Scharlemann: Producing reducible 3-manifolds by surgery on a knot, Topology 29 (1990) $481-500$.

[11] E. Starr: Curves in handlebodies, Thesis UC Berkeley (1992).

[12] Y-Q. Wu: Incompressibility of surfaces in surgered 3-manifold, Topology 31 (1992) 271-279.

[13] Y-Q. Wu: D-reducing Dehn surgeries and 1-bridge knots, Math. Ann. 295 (1993) 319-331.

Department of Mathematics

University of lowa

lowa City, IA 52246

E-mail: wu@math.uiowa.edu 